\title{
Strength theories of disjunctive visual detection'
}

WAYNE A. WICKELGREN

MASSACHUSETTS INSTITUTE OF TECHNOLOGY

Zero, 1,2, or 3 black dots are tachistoscopically presented on a white field. There are two altemative tasks: (a) to decide on the presence of each of the left, middle, and right dots (multiple detection) or (b) to decide whether any of the dots was present (disjunctive detection). The results indicate that in disjunctive detection, $S$ s do not add together the strengths of the three dot positions and compare this sum to a criterion. Rather they combine their decisions about each dot, responding "yes" to the array, if and only if they decide "yes" to any one dot. Strength distributions appear to be invariant with respect to irrelevant stimuli. Invariance with respect to report order holds approximately. However, dots reported on first are slightly more detectable. This suggests a successive scanning process, whose rate is independent of whether a stimulus is present or absent at the position scanned.

According to the criterion decision rule of signal detection theory, the physical dimension of "stimuluspresent, stimulus-absent" maps into some single psychological dimension, which will be called stimulus "strength." The mapping is noisy, so a particular value on the physical dimension (either "present" or "absent" in the simplest case) maps into a distribution of values on the (psychological) strength dimension. It is almost always assumed that the strength distributions obtained under this noisy mapping are unimodal.

More particularly, it is assumed that they are normal distributions, though about the only property of the normal distribution that is testable in most experiments is the unimodal property. Fortunately, it is of little concern what unimodal distribution function we assume, since at our present level of predictive precision, any unimodal distribution function is an adequate approximation to any other unimodal distribution function. The normal distribution lacks an analytic solution for its integral, but, in most cases, this disadvantage is more than offset by the convenient property that sums of normally distributed random variables are themselves normally distributed.

Thus, at present, what we are assuming is that we can characterize the internal representative of the physical dimension (present-absent) on a unidimensional (strength) scale, with a value on the physical dimension mapping into a central-tendency measure (mean) and a spread measure (standard deviation) for the corresponding unimodal (normal) strength distribution. The mean of the strength distribution for the stimulus-present condition is assumed to be greater than or equal to the mean of the strength distribution for the stimulus-absent condition.
According to the criterion rule, a subject decides whether the stimulus was present or absent on a given trial by comparing the stimulus strength with a criterion, or cut-off, on the strength scale. If the strength exceeds the criterion, he responds "yes" (stimulus was present); otherwise, he responds "no." The criterion placement may be affected by quite a number of factors, but it is essential to assume that it is not a function of the strength of the stimulus on that trial. Thus, the probability of a "yes" response in a given condition is the area under the strength distribution for that condition to the right of the criterion. Hence, the empirical probability of a "yes" response in the "stimulus-present" condition locates the mean of the strength distribution for this condition with respect to the criterion in units of the standard deviation of this strength distribution.

Similarly, the empirical probability of a "yes" response in the "stimulus-absent" condition locates the mean of the strength distribution for this condition with respect to the criterion in units of the standard deviation of this strength dimension. The criterion for the stimulus-absent condition is assumed to be the same as the criterion for the stimuluspresent condition. If one assumes the two standard deviations to be equal or determines their ratio by means of an operating characteristic (Swets, Tanner, \& Birdsall, 1961), one can determine the distance (d') between the means of the two strength distributions (in units of one of the two standard deviations), since one knows the distance of each mean from the common criterion.

The present study is concerned with detection of several simultaneously presented visual stimuli. The basic experiment is as follows: On each trial an $S$ is presented with a white field on which there may be $0,1,2$, or 3 black dots. Dots $\left(1 / 4^{\circ}\right.$ in diameter $)$ can appear at only three positions, left, middle, and right. The positions are widely separated $\left(1-1 / 4^{\mathrm{O}}\right.$ from center to center) and on a horizontal line. There are two basic decision sets: (a) to make separate decisions about the presence or absence of the left, middle, and right dots and (b) to make one decision as to whether any $(1,2$, or 3$)$ of the three dots was present. The first decision set will be called multiple detection and the second decision set will be called disjunctive detection.

The primary purpose of the present study is to determine if disjunctive detection can be predicted from multiple detection, and, if so, what the law is. Thus, the present study is primarily concerned with the decision procedure used by the $S$ to transform 
several simultaneous visual stimuli into responses, rather than with the nature of the representation of visual stimuli in the visual system.

Presumably, we shall find that the criterion decision rule of signal detection theory provides an adequate description of the detection of each of the three dots under the various conditions of the multiple detection task. However, it is necessary to verify this, and the verification will slightly extend the range of applicability of the criterion rule.

In conjunction with determining the applicability of the criterion rule to multiple detection of simultaneous visual stimuli, any interfering effects of the presentation of one stimulus on the detectability of another stimulus can be noted. Also, the effects of order of report on detectability of simultaneous visual stimuli will be studied.

However, the primary focus of the study will be on determining the decision rule for disjunctive detection, assuming that the strength distributions for each dot (under each condition of presentation of the other dots) are identical in both multiple and disjunctive decision procedures. There are two primary possibilities for the disjunctive decision procedure: (a) the additive strength rule and (b) the multiplicative probability rule.

The additive strength rule assumes that the $S$ adds the stimulus strength value of each of the three dot positions and compares this sum to a criterion, responding "yes" (at least one of the dots was present), if, and only if, the sum exceeds the criterion. This is a very good decision rule in the sense that it loses none of the information in the stimulus strength' before the final decision. Perhaps partly for this reason, it has been the decision rule assumed by signal detection theorists for a task very similar to the disjunctive detection task, namely, the multiple-look detection task (Swets, Shipley, McKey, \& Green, 1959; also see Luce, 1963, p. 136). The results of a multiple-look auditory detection task with successive "looks" were generally supportive of the additive strength rule (Swets, Shipley, McKey, \& Green, 1959). Naturally, this does not mean that the additive strength rule will necessarily hold for disjunctive visual detection of simultaneous stimuli.

The multiplicative probability rule assumes that in disjunctive detection the $\mathrm{S}$ first makes a decision about the presence or absence of each of the three dots, as in multiple detection, and then combines these decisions into an overall decision. Decisions are being combined, rather than strengths. If decisions are being combined, the obvious rule is to respond "yes" to the entire stimulus, if and only if you decided "yes" with respect to at least one of the three dots. Thus, one is predicting the probability of a "yes" response in the disjunctive decision task from the probabilities of "yes" responses to $L, M$, and $R$ dots when the dots are present and when they are absent in the multiple detection task.
As stated thus far, the multiplicative probability rule does not appear to require analysis of multiple detection in terms of the criterion rule of signal detection theory. However, there is no reason to be sure that the criterion for "yes" decisions to each dot will be identical for disjunctive and multiple detection tasks. Thus, it may be necessary to use the $d^{\prime}$ values obtained by the criterion-rule analysis of the multiple detection task, in conjunction with estimates of the criterion shifts from multiple to disjunctive detection tasks, to determine the probabilities of "yes" decisions to each dot in the disjunctive detection task.

Several previous studies either tested or assumed the validity of the multiplicative probability rule for disjunctive visual detection of two dots to different locations on the same eye (Bouman \& van den Brink, 1952; van den Brink \& Bouman, 1954) or to corresponding locations on the two eyes (Pirenne, 1943; Casperson \& Schlosberg, 1950; Collier, 1954; Matin, 1962). These studies were not done from the perspective of signal detection theory, and they have used an inadequate formulation of the multiplicative probability rule. The inadequate formulation is that the probability of not detecting either of two stimuli when both are presented is the product of the probabilities of not detecting each stimulus when it is presented alone. The S's set is for disjunctive detection in both cases, and he makes only one "yes-no" decision. All that differs is the stimuli that are presented. What is wrong with this analysis is that it ignores the probability of false detection (responding "yes" to a point when nothing was presented there), implicitly assuming it to be zero for each point.

Naturally, these studies also do not determine the disjunctive false detection rate (probability of a "yes" response, when neither stimulus is presented). But even if they did include a disjunctive false detection condition, it would be impossible to predict the binary disjunctive detection probability for one condition (e.g., both stimuli presented) from the binary disjunctive detection probabilities for all the other conditions, except under the restrictive condition that the correct detection probabilities for each stimulus be equal and also that the false detection probabilities for each stimulus be equal. The reason is that for each stimulus position there are two parameters to be estimated, the correct and false detection rates, making four parameters to be estimated for binary disjunctive detection, and there are only four independent statistics of the data, the probabilities of disjunctive detection in each of the four possible conditions of stimulus presentation. For ternary disjunctive detection the number of independent statistics of the data begins to outrun the number of parameters to be estimated, eight to six. Still, it would be a much more powerful test of the multiplicative probability rule to estimate the parameters from a different task (e.g., multiple detection) and use 
them to predict the probabilities of disjunctive detection in all the various conditions, even if a few criterion-shift parameters had to be estimated.

\section{METHOD}

\section{Subjects}

Ss were three paid volunteers who were students at M.I.T.

\section{Apparafus}

A five-field tachistoscopic viewing box was used, providing front illumination of visual fields $11-1 / 2^{\circ}$ square (two going to the right eye, two to the left eye, and one to both eyes). The light sources were cold-cathode mercury argon lamps coated with magnesium tungstate phosphor. Timing was done by a Sky Instrument Company type tachistoscopic programmer. A chin rest was used to reduce head movement.

\section{Procedure}

The experiment was done under largely dark adapted conditions, though only about $3 \mathrm{~min}$ of dark adaptation preceded the beginning of an experimental session. A trial consisted of several sec of viewing a dark field with a dim red fixation light $\left(1 / 4^{\circ}\right.$ in diameter) in the center of the field, followed by the $S$ pressing a button when he was ready for the stimulus field, followed in $.5 \mathrm{sec}$ by a $7 \mathrm{msec}$ stimulus field containing any combination of $0,1,2$, or 3 black dots on a white field, followed by the S's decision concerning the presence of dots on the stimulus field, followed in 10 to $15 \mathrm{sec}$ by the next trial. Between trials the fleld was dark except for the fixation light.

The three possible dots were $1 / 4^{\circ}$ in diameter, oriented on a horizontal line with $1-1 / 2^{\circ}$ between the centers of adjacent dots. The center of the middle dot was located $1 / 2^{\circ}$ directly above the center of the red fixation light. The black dots had a reflectance of about $2 \%$, and the white background had a reflectance of about $90 \%$. Kodak neutral density filters were used to set the level of illumination reaching the $S$ at an intermediate level of detectability of the dots. The illumination of the blank white card was about .016 ft- $\mathrm{L}$ for Ss IO and JM and about .024 ft-L for S BW, measured at the eyepiece with a Macbeth illuminometer.

\section{Design}

There were eight different stimulus conditions: no dots $(N)$, left dot only (L), middle dot only (M), right dot only (R), left and middle dots (LM), middle and right dots (MR), left and right dots (LR), and all three dots (LMR). Stimulus conditions were presented randomly in blocks of 10 trials, all conditions being presented once each, except $\mathrm{N}$ which appeared three times per block. Ss received 12 blocks, or 120 experimental trials, per $(1-h)$ session. There were six practice trials at the beginning of each session, followed by seven blocks, followed by a 5 -min rest, followed by two practice trials, followed by five more blocks.

In any given session Ss had one of three possible decision sets: (LMR) multiple detection, deciding about the presence of each dot in left to right order, (RML) multiple detection, deciding about the presence of each dot in right to left order, and (D) disjunctive detection, deciding whether any of the dots was present. Two or three sessions were required to determine a suitable level of illumination, followed by one more practice session with each decision set, followed by 10 sessions with each of the three decision sets, randomly ordered in blocks of three sessions. Thus, there were 35 to $361-h$ sessions for each $S$ in the experiment, yielding 120 trials in every stimulus condition for each decision set, except that there were 360 trials in the no-dot $(\mathrm{N})$ stimulus condition for each decision set.

In addition to their "yes-no" decisions in both tasks, Ss were required to state confidence in each "yesno" decision on a three-point rating scale $(3$ representing most confidence, 1 representing least confidence). Ss were urged to use all confidence categories approximately equally often. They were also instructed to use the practice sessions to determine their standards for each response and thereafter to try to maintain these standards without changing them. In this way it was hoped, criterion variance would be reduced.

\section{STRENGTH THEORY OF MULTIPLE ANO DISJUNCTIVE DETECTION}

\section{Definitions}

(1) Let $s_{x}(y, z)$ be the strength in the visual system (the degree of activation of the internal representative) of dot position $x$ under stimulus condition $y$ and report condition $z$. In the present experiment $x$ can be $L, M$, or $R$; y can be $N, L, M, R, L M, M R, L R$, or LMR; $z$ can be LMR or RML for the multiple task and $\mathrm{D}$ for the disjunctive task.

(2) Let $c_{x}(y, z)$ be the criterion for a "yes" decision to dot position $\mathrm{x}$ under stimulus condition $\mathrm{y}$ and report condition $\mathbf{z}$.

(3) Let $s_{L M R}(y, z)=s_{L}(y, z)+s_{M}(y, z)+s_{R}(y, z)$.

(4) Let $c_{\text {LMR }}(y, D)$ be the criterion for a "yes" response in the disjunctive task (under the additive strength rule).

(5) Let $p_{x}(y, z)$ be the probability of a "yes" decision to dot position $\mathbf{x}$ under stimulus condition $\mathrm{y}$ and report condition $\mathbf{z}$.

(6) Let $p_{\text {LMR }}(y, D)$ be the probability of a "yes" decision in the disjunctive decision task.

\section{Criterion Decision Rule for Multiple Defection}

Respond "yes" to dot position $x$ under stimulus condition $y$ and report condition $z=L M R$ or $R M L$, iff $s_{x}(y, z)-c_{x}(y, z) \geq 0$. It is also assumed that the 
random variable $s-c$ is normally distributed: $s_{x}(y, z)-$ $c_{x}(y, z) \sim N\left[\mu_{x}(y, z)-\mu_{c x}(y, z),\left(\sigma_{x}{ }^{2}(y, z)+\sigma_{c x}{ }^{2}\right.\right.$ $(y, z))^{1 / 2}$.

This implies that: $p_{x}(y, z)=\int_{0}^{\infty} N\left[\mu_{x}(y, z)-\mu_{c x}\right.$ $\left.(y, z),\left(\sigma_{x}^{2}(y, z)+\sigma_{c x}{ }^{2}(y, z)\right)^{1 / 2}\right]$.

\section{Alternative Decision Rules for Disjunctive Detection}

(1) (Additive Strength Rule) Respond "yes" to the entire array, iff $s_{L M R}(y, D)-c_{L M R}(y, D) \geq 0$.

This implies that: $p_{L M R}(y, D)=\int_{0}^{\infty} N\left[\mu_{L}(y, D)+\right.$ $\mu_{M}(y, D)+\mu_{R}(y, D)-\mu_{c}(y, D),\left(\sigma_{L}{ }^{2}(y, D)+\sigma_{M}{ }^{2}(y, D)\right.$ $\left.+\sigma_{\mathbf{R}}^{2}(\mathrm{y}, \mathrm{D})+\sigma_{\mathrm{c}}^{2}(\mathrm{y}, \mathrm{D})\right)^{1 / 2}$

(2) (Multiplicative Proability Rule) Respond "yes" to the entire array, if you decided that at least one of the dots was present, i.e., $\max \left\{\beta_{X}(y, D)-c_{x}(y, D)\right\}$ $\geq 0$, where $x=L, M$, or $R$.

This implies that: $p_{L M R}(y, D)=1-\left[1-p_{L}(y, D)\right]$. $\left[1-p_{M}(y, D)\right] \cdot\left[1-p_{R}(y, D)\right]$.

\section{Strength Invariance Assumptions}

(1) (Invariance with respect to Decision set and Report Order) $s_{x}(y)=s_{x}(y, L M R)=s_{x}(y, R M L)=$ $s_{x}(y, D)$ for all $x$ and $y$.

(2) (Invariance with respect to Irrelevant Stimuli) $s_{x}(y)=s_{x}$, if $y$ ineludes presentation of a dot in position $x ; s_{x}(y)=n_{x}$, if $y$ does not include presentation of a dot in position $x$, for all $y$.

(3) (Invariance of Noise with respect to Position $\mathbf{n}=\mathbf{n}_{\mathrm{L}}=\mathbf{n}_{\mathbf{M}}=\mathbf{n}_{\mathbf{R}}$.

In this paper, to say that two random variables are equal means that they have the same distribution function.

Thus, the present experiment is assumed to involve only four different strength distributions: $s_{L} \sim N\left[\mu_{L}\right.$, $\left.\sigma_{\mathbf{L}}\right], \mathbf{s}_{\mathbf{M}} \sim \mathbf{N}\left[\mu_{\mathbf{M}},{ }^{\sigma_{\mathbf{M}}}\right], \mathbf{s}_{\mathbf{R}} \sim \mathbf{N}\left[\mu_{\mathbf{R}},{ }^{\sigma_{\mathbf{R}}}\right]$, and $\mathrm{n} \sim \mathrm{N}$ $[0,1]$. Since measurement of strengths is on an interval scale, we can arbitrarily choose the mean and standard deviation of one strength distribution to be 0 and 1 , respectively.

The primary purpose of the present study is to test the two decision rules for disjunctive detection in conjunction with the part of the first invariance assumption that assumes the same strength distributions apply in both multiple and disjunctive detection. The second invariance assumption is not necessary for this primary purpose, but is moderately interest- ing in its own right, and testable in this study. The third invariance assumption is irrelevant to the primary purpose, and untestable by present methods, but it simplifies computation, and has no effect on any predictions for the present experiment.

\section{Criterion Invariance Assumptions}

Criterion invariance assumptions are not necessary to test the validity of the two decision rules for disjunctive detection, and criterion invariance over stim- ulus and report conditions is particularly likely not to hold. It is safest to assume that $c_{x}(y, z)$ may be different for every different $(x, y, z)$. Fortunately, operating characteristics allow us to eliminate the effects of different criteria and obtain empirical estimates of strengths, although by this means we can only measure strength differences, not absolute strength levels.

However, it is necessary to make one type of criterion invariance assumption. We must assume that $c_{R}(R, z)=c_{R}(N, z), c_{L}(L M, z)=c_{L}(M, z), c_{M}$ $(L M R, z)=c_{M}(L R, z)$, and all similar assumptions. Specifically, we must assume that the criterion for a particular dot position does not depend upon whether a dot was actually presented in that position, when we hold constant the stimulus conditions for the other two dot positions and the report condition. An $S$ cannot have a different criterion for the dot-present and dot-absent conditions at a particular location, prior to detecting whether a dot is there or not. This is an absolutely basic assumption for the testing of signal detection theory in any context, and it is possible to imagine ways in which it might be false, but the assumption seems completely safe in the present instance.

\section{Operating Characteristics and Strength Measurement}

An operating characteristic in multiple detection is a plot of $p_{x}(y, z)$ against $p_{x}(w, z)$ for a pair of stimulus conditions $y$ and $w$ that differ only in that $y$ has a dot in position $x$ and $w$ does not. These are precisely the conditions under which we assume that $c_{x}(y, z)=c_{x}(w, z)$. In disjunctive detection $(x=L M R)$, it is assumed that $c_{L M R}(y, D)=c_{L M R}(w, D)$ for all $y$ and $w$, and thus $p_{L M R}(y, D)$ is plotted against $p_{\text {LMR }}(\mathrm{N}, \mathrm{D})$ for all $\mathrm{y} \neq \mathrm{N}$.

If only "yes-no" decisions were elicited, each operating characteristic would consist of one point. However, by means of the confidence judgment technique, a number of $\left[p_{x}(w, z), p_{x}(y, z)\right]$ pairs are generated, one pair for each different criterion, $c_{x}$ $(w, z)=c_{x}(y, z)$, generated by the confidence judgment method.

Explanations of the confidence judgment method and the operating characteristics it generates are to be found in many other places (e.g., Egan, Schulman, \& Greenberg). For the present paper, the properties of operating characteristics will be stated without proofs; the proofs can be found elsewhere (e.g., Green \& Swets, 1966). The principal properties are:

(1) The fit of an operating characteristic to a straight line, when plotted on normal-normal probability coordinates, is a measure of the adequacy of the normal distribution assumptions for the random variables.

(2) On the normal-normal plot of $p_{x}(y, z)$ on the vertical axis vs $p_{x}(w, z)$ on the horizontal axis, the horizontal intercept of the best-fitting straight line 
is $d_{x}{ }^{\prime}(y, w, z)=\mu_{x} /\left(1+\sigma_{c x}\right)^{1 / 2}$, and the slope of the best-fitting line is $b_{x}(y, w, z)=\left(1+\sigma_{c x^{2}}\right)^{1 / 2} /$ $\left(\sigma_{x^{2}}+\sigma_{c x^{2}}\right)^{1 / 2}$.

\section{Testing the Invariance Assumptions}

We can rather directly test the invariance of strengths over the two multiple-detection report orders, LMR and RML, by observing whether $d_{x}{ }^{\prime}(y, w, L M R)=$ $d_{x}{ }^{\prime}(y, w, R M L)$ for all $x, y$, and $w$.

Invariance with respect to irrelevant stimuli is also rather directly testable in the multiple detection task by observing whether:

$$
\begin{aligned}
d_{L^{\prime}}{ }^{\prime}(L, N, z) & =d_{L}{ }^{\prime}(L M, M, z)=d_{L}{ }^{\prime}(L R, R, z) \\
& =d_{L^{\prime}}{ }^{\prime}(L M R, M R, z)=d_{L}{ }^{\prime} \\
& =\mu_{L} /\left(1+\sigma_{c L} 2\right)^{1 / 2} \\
d_{M}{ }^{\prime}(M, N, z) & =d_{M}{ }^{\prime}(L M, L, z)=d_{M}{ }^{\prime}(M R, R, z) \\
& =d_{M}{ }^{\prime}(L M R, L R, z)=d_{M}{ }^{\prime} \\
& =\mu_{M} /\left(1+\sigma_{c M} 2\right)^{1 / 2}
\end{aligned}
$$

and

$$
\begin{aligned}
d_{R}^{\prime}(R, N, z) & =d_{R}^{\prime}(M R, M, z)=d_{R}{ }^{\prime}(L R, L, z) \\
& =d_{R}^{\prime}(L M R, L M, z)=d_{R}{ }^{\prime} \\
& =\mu_{R} /\left(1+\sigma_{c R}{ }^{2}\right)^{1 / 2}
\end{aligned}
$$

for $z=L M R$ and $R M L$.

Testing the Additive Strength Rule for Disjunctive Detection According to the additive strength rule,

$$
\begin{aligned}
& d^{\prime}(L)=d_{\text {LMR }}(L, N, D)=\mu_{L} /\left(3+\sigma_{c}^{2}\right)^{1 / 2} \\
& d^{\prime}(M)=d_{L M R}(M, N, D)=\mu_{M} /\left(3+\sigma_{C}{ }^{2}\right)^{1 / 2} \\
& d^{\prime}(R)=d_{L M R}(R, N, D)=\mu_{R} /\left(3+\sigma_{c}{ }^{2}\right)^{1 / 2} \\
& \left.d^{\prime}(L M)=d_{L M R}(L M, N, D)=\left(\mu_{L}+\mu_{M}\right) /\left(3+\sigma_{C}\right)^{2}\right)^{1 / 2} \\
& d^{\prime}(M R)=d_{L M R}(M R, N, D)=\left(\mu_{M}+\mu_{R}\right) /\left(3+\sigma_{c}{ }^{2}\right)^{1 / 2} \\
& d^{\prime}(L R)=d_{L M R}(L R, N, D)=\left(\mu_{L}+\mu_{R}\right) /\left(3+\sigma_{c}\right)^{1 / 2} \\
& d^{\prime}(L M R)=d_{L M R}(L M R, N, D) \\
& =\left(\mu_{L}+\mu_{M}+\mu_{R}\right) /\left(3+\sigma_{c}\right)^{2 / 2}
\end{aligned}
$$

Thus, a very simple test of the additive strength rule can be made strictly within the context of disjunctive detection by observing whether $d^{\prime}(L M)=d^{\prime}(L)+$ $d^{\prime}(M), \quad d^{\prime}(M R)=d^{\prime}(M)+d^{\prime}(R), \quad d^{\prime}(L R)=d^{\prime}(L)+d^{\prime}(R)$, and $d^{\prime}(L M R)=d^{\prime}(L)+d^{\prime}(M)+d^{\prime}(R)$. If the data pass this test, a further test of the additive strength rule can be made by determining if the $d$ and $b$ values for disjunctive detection can be predicted from the $d$ and $b$ values for multiple detection. This requires estimation of criterion variance parameters and an hypothesis about the relationship between the criterion for disjunctive detection and the criteria for multiple detection.

\section{Testing the Multiplicative Probability Rule for Disjunctive Detection}

If we could be sure that the criterion for detecting each dot was identical for all report conditions, in particular that $p_{x}(y, D)=p_{x}(y, z)$ for $z=L M R$ or $R M L$, then testing the multiplicative probability rule would be extremely simple. All we would have to do is determine whether: $p_{L M R}(y, D)=1-\left[1-p_{L}(y, z)\right] \cdot[1-$ $\left.p_{M}(y, z)\right] \cdot\left[1-p_{R}(y, z)\right]$, for $z=L M R$ or RML. In general, however, it is necessary to estimate 1,2 , or 3 criterion-shift parameters to determine how much the criterion for detecting each dot has changed from multiple to disjunctive detection. It is quite reasonable for the criteria to change, since the frequency of "no" responses would be much reduced in disjunctive detection from multiple detection, if the criteria did not change. If Ss are trying to keep their frequencies of "yes" and "no" responses not too discrepant, a criterion shift would be in order.

Allowing for the possibility of a criterion shift means that we use the $d^{\prime}$ values and the criterion values from the multiple detection task for each condition and estimate one criterion-shift parameter for each dot position, with this criterion shift applying to the criterion for that dot position under every stimulus condition. This means that whatever differences there are in the criteria for the same dot position under different stimulus conditions in the multiple detection task, these differences are assumed to be preserved by the criterion shift to the disjunctive detection task.

\section{Operating Characteristics}

\section{RESULTS AND DISCUSSION}

In accordance with the normal distribution assumption of signal detection theory, the operating characteristics were reasonably well fit by straight lines on normal-normal probability coordinates. However, out of a maximum of five points on an operating characteristic, there were usually only three or four distinct points, often only two, and occasionally only one point. Obviously, in the latter two cases, it is impossible to test the normal distribution assumption. When there are only three points, the points must be fairly widely spaced in order to provide a reasonable test of the normal distribution assumption. Fortunately, when there were three or four points, they were almost always well spaced, and about two-thirds of the operating characteristics could be used to test the normal distribution assumption. These cases gave no reason to reject the assumption, as deviations were generally small and certainly not systematic.

Slopes of the operating characteristics were very consistently less than unity, definitely rejecting the assumption that the variance of dot-present and dotabsent conditions are equal. Slopes less than unity imply that the variances of the strength distributions for the dot-present conditions are greater than the variances of the strength distribution for the dotabsent conditions. Also, the slopes tend to be negatively correlated with $d^{\prime}$, though there is considerable variability. This is just what has been found in other studies of visual signal detection (Swets, Tanner, \& 
Birdsall, 1961; Nachmias \& Steinman, 1963), except that in the present instance the dots are dark on a light background, and in the previous studies the stimuli to be detected were light on a dark background.

What incorporates these results into a common framework is that the variance of a strength distribution increases with its mean, and in the present instance the strength dimension is darkness, whereas in the previous two studies it was lightness. The present results suggest that darkness and lightness are two psychological dimensions, rather than two ends of the same dimension with complete darkness being simply zero lightness. Other psychological studies indicate that it is the contrast between the stimulus and the ground which is the primary internal representation for visual intensity, and neurophysiological studies indicate that there are cells responding to dark centers on light grounds as well as light centers on dark grounds. Thus, there is hardly anything startling about the present slope findings.

\section{Strength Invariance Assumptions}

Table 1 presents the $d^{\prime}$ and $b$ (slope) parameters for the (visually) best-fitting straight lines for each operating characteristic in the multiple detection task. Where there was only one distinct point on the operating characteristic (because Ss did not use more than one degree of confidence with any frequency), the slope, naturally, cannot be computed. In these cases, $d^{\prime}$ was estimated by using the average slope for all the other operating characteristics for that $S$, rather than by assuming the slope to be unity.

Invariance with respect to irrelevant stimuli seems to hold for the present experiment. There is quite a little variability in the $d$ ' values for detection of any particular dot by any particular $s$, but there is no systematic tendency for d'values to be lowered or increased by the presence of the other dots in the visual field, even though these dots have to be detected also.
Table 2. Values of $d^{\prime}$ and $b$ for disjunctive detection operating characteristics

\begin{tabular}{lcccccc} 
Stimulus & \multicolumn{2}{c}{$J M$} & \multicolumn{2}{c}{$B W$} & \multicolumn{2}{c}{10} \\
Condition vs. N & $d^{\prime}$ & $b$ & $d^{\prime}$ & $b$ & $d^{\prime}$ & $b$ \\
\hline$L$ & 1.70 & .9 & 1.52 & .8 & 1.28 & .7 \\
$M$ & 1.48 & 1.0 & 1.33 & .8 & 2.07 & .5 \\
$R$ & 1.15 & .8 & .50 & 1.0 & .53 & .7 \\
LM & 1.83 & 1.3 & 2.36 & .6 & 2.46 & .6 \\
MR & 1.69 & 1.2 & 1.34 & .8 & 2.45 & .5 \\
LR & 1.48 & .9 & 1.55 & .8 & 1.61 & .7 \\
LMR & 2.29 & .8 & 2.34 & .6 & 2.47 & .6 \\
\hline
\end{tabular}

There is a small, but systematic, tendency for d' values to depend on report order in multiple detection, presumably because report order dictates the order of some sort of sequential visual scanning operation. The direction of the effect on $d^{\prime}$ is what one would expect, namely, slightly lower $d^{\prime}$ values for a dot position scanned last, rather than first. Presumably in the time taken to scan other dot positions, the visual very short-term memory trace for a dot position decays somewhat. The invariance with respect to irrelevant stimuli suggests that scanning rate is not appreciably affected by the presence or absence of a dot at the position scanned.

However, the effect of report order on d' is relatively small, so one must be cautious in drawing any definite conclusions about visual scanning operations from it. If there is sequential visual scanning, it must be quite rapid in the present experiment, in comparison to the rate of decay of the visual memory trace. As a first approximation, one could certainly assume both invariance with respect to irrelevant stimuli and invariance with respect to report order, for the present multiple detection task.

\section{Additive Strength Rule for Disjunctive Detection}

Table 2 presents the $d^{\prime}$ and $b$ values for the disjunctive detection task. One conclusion is immediately obvious. The additive strength rule is false for dis-

Table 1. Values of $d^{\prime}$ and $b$ (slope) for multiple-detection operating characteristics.

\begin{tabular}{|c|c|c|c|c|c|c|c|c|c|c|c|c|c|}
\hline \multirow{3}{*}{$\begin{array}{l}\text { Stimulus } \\
\text { Condition } \\
\text { with } \\
\text { L dot }\end{array}$} & \multirow{3}{*}{$\begin{array}{l}\text { Stimulus } \\
\text { Condition } \\
\text { with } \\
\text { No L dot }\end{array}$} & \multicolumn{4}{|c|}{$J M$} & \multicolumn{4}{|c|}{ BW } & \multicolumn{4}{|c|}{10} \\
\hline & & \multicolumn{2}{|c|}{ LMR } & \multicolumn{2}{|c|}{ RML } & \multicolumn{2}{|c|}{ LMR } & \multicolumn{2}{|c|}{ RML } & \multicolumn{2}{|c|}{ LMR } & \multicolumn{2}{|c|}{ RML } \\
\hline & & $d^{\prime}$ & $b$ & $d^{\prime}$ & $b$ & d' & $b$ & $d^{\prime}$ & $b$ & $d^{\prime}$ & $b$ & $d^{\prime}$ & $b$ \\
\hline L & $N$ & 2.06 & .7 & 2.55 & - & 2.01 & .6 & 1.55 & .7 & 1.69 & .6 & 1.94 & .5 \\
\hline LM & $M$ & 2.16 & .6 & 1.98 & .8 & 1.84 & .8 & 2.29 & .8 & 2.20 & .5 & 1.88 & .3 \\
\hline LR & $\mathrm{R}$ & 2.35 & .6 & 2.20 & .5 & 2.06 & .6 & 1.47 & .8 & 2.53 & .3 & 2.24 & .4 \\
\hline LMR & MR & 2.94 & .5 & 2.35 & .4 & 2.12 & .6 & 1.94 & .6 & 2.53 & .4 & 1.48 & .6 \\
\hline$M$ dot & No $M$ dot & & & & & & & & & & & & \\
\hline$M$ & $N$ & 2. 14 & .5 & 1.59 & .9 & 1.47 & .6 & 1.36 & .8 & 2.35 & .4 & 1.81 & .8 \\
\hline LM & $L$ & 1.58 & .7 & 2.07 & .7 & 1.28 & .7 & 1.20 & .7 & 1.74 & .4 & 2.15 & .5 \\
\hline MR & $R$ & 1.88 & .6 & 1.80 & .8 & .92 & .6 & 1.61 & .6 & 1.56 & .6 & 2.00 & .5 \\
\hline $\begin{array}{l}\text { LMR } \\
\mathrm{R} \text { dot }\end{array}$ & $\begin{array}{l}\text { LR } \\
\text { No } R \text { dot }\end{array}$ & 2.25 & .5 & 2.47 & .4 & 1.55 & .6 & 1.23 & .8 & 1.95 & .3 & 1.78 & .5 \\
\hline $\mathrm{R}$ & $N$ & 2.35 & - & 2.23 & - & 1.11 & .8 & .69 & .7 & .64 & .6 & 1.16 & .5 \\
\hline MR & $M$ & 1.98 & .5 & 2.22 & .6 & .99 & .8 & 1.10 & .8 & .67 & .6 & .68 & .4 \\
\hline LR & $L$ & 2.55 & .5 & 2.59 & .6 & .56 & .7 & .64 & .8 & 1.16 & .5 & .93 & .6 \\
\hline LMR & LM & 2.25 & .6 & 1.70 & .7 & .57 & .7 & .67 & .8 & .58 & .5 & .90 & .7 \\
\hline
\end{tabular}


Table 3. Percentage of "yes" responses in multiple and disjunctive detection

\begin{tabular}{|c|c|c|c|c|c|c|c|c|c|}
\hline$S$ & $\begin{array}{l}\text { Stimulus } \\
\text { Condition }\end{array}$ & $\begin{array}{r}{ }^{P} \\
\text { LMR }\end{array}$ & RML & LMR & & LMR & RML & $\begin{array}{l}{ }^{P D} \\
D\end{array}$ & $\begin{array}{c}\mathrm{PD}_{\mathrm{D}} \\
\text { Predicted }\end{array}$ \\
\hline \multirow[t]{8}{*}{$J M$} & $\mathrm{~N}$ & 11 & 7 & 17 & 19 & 6 & 8 & 26 & 24 \\
\hline & $\mathrm{L}$ & 73 & 74 & 18 & 19 & 6 & 8 & 80 & 76 \\
\hline & $\bar{M}$ & 7 & 18 & 71 & 76 & 5 & 7 & 78 & 76 \\
\hline & $\mathrm{R}$ & 7 & 8 & 22 & 22 & 69 & 69 & 65 & 60 \\
\hline & $L M$ & 68 & 82 & 69 & 85 & 6 & 8 & 93 & 94 \\
\hline & MR & 6 & 7 & 75 & 78 & 57 & 65 & 90 & 86 \\
\hline & LR & 69 & 66 & 15 & 14 & 68 & 74 & 78 & 84 \\
\hline & LMR & 76 & 69 & 73 & 72 & 65 & 61 & 91 & 95 \\
\hline \multirow[t]{8}{*}{ BW } & $N$ & 21 & 11 & 29 & 28 & 17 & 31 & 34 & 39 \\
\hline & $L$ & 80 & 62 & 13 & 22 & 17 & 32 & 82 & 73 \\
\hline & $M$ & 15 & 7 & 73 & 72 & 14 & 12 & 78 & 76 \\
\hline & $R$ & 27 & 13 & 17 & 19 & 55 & 56 & 51 & 53 \\
\hline & $L M$ & 76 & 73 & 52 & 62 & 10 & 19 & 86 & 87 \\
\hline & MR & 14 & 10 & 49 & 68 & 47 & 49 & 79 & 72 \\
\hline & $L R$ & 77 & 63 & 11 & 21 & 38 & 56 & 79 & 76 \\
\hline & LMR & 72 & 67 & 60 & 64 & 31 & 45 & 88 & 88 \\
\hline \multirow[t]{8}{*}{10} & $\mathrm{~N}$ & 20 & 9 & 17 & 21 & 7 & 7 & 30 & 36 \\
\hline & $L$ & 69 & 61 & 8 & 14 & 3 & 8 & 69 & 71 \\
\hline & $M$ & 6 & 2 & 71 & 78 & 6 & 3 & 79 & 77 \\
\hline & $\mathbf{R}$ & 9 & 4 & 22 & 22 & 29 & 43 & 49 & 56 \\
\hline & $L M$ & 62 & 49 & 58 & 71 & 5 & 7 & 88 & 85 \\
\hline & $M R$ & 4 & 6 & 67 & 75 & 30 & 33 & 84 & 81 \\
\hline & $L R$ & 64 & 58 & 11 & 12 & 37 & 38 & 74 & 78 \\
\hline & LMR & 60 & 48 & 61 & 62 & 32 & 32 & 88 & 88 \\
\hline
\end{tabular}

junctive detection of widely spaced dots. $d^{\prime}(L M R) \neq d^{\prime}(L)$ $+d^{\prime}(M)+d^{\prime}(R)$ for any of the three Ss, nor do any of the other equalities predicted by the additive strength rule hold. Not only are the mispredictions reasonably large in size, but they are all off in the same direction, namely, predicting too much improvement in disjunctive detection when the number of dots is increased.

\section{Multiplicative Probability Rule for Disjunctive Detection}

Table 3 presents the empirical probability of a "yes" response in every multiple and disjunctive detection condition, along with a predicted probability of a "yes" response in each disjunctive detection condition. The latter prediction is made using the multiplicative probability rule to predict the probability of disjunctive detection from the probabilities of detecting each of the three dots in the corresponding multiple detection condition (averaged over LMR and RML). For S IO, this could be done directly, using the probability data for multiple detection given in Table 3 . For the other two Ss, criterion shift parameters were estimated for both $L$ and $R$ dots for each $S$, and applied to the probabilities in Table 3 to obtain adjusted probabilities. In making this adjustment, the difference in standard deviation of the dot-present and dot-absent conditions was taken into account. All four estimated criterion shifts are in the direction of an $S$ requiring greater strength in order to decide he saw a dot at an $\mathrm{L}$ or $\mathrm{R}$ position in the disjunctive detection task than in the multiple detection task. This would tend to more nearly equate the frequency of "yes" and "no" responses in the disjunctive detection task than would a maintenance of the criteria used in the multiple detection task. Thus, the criterion shifts are all in the intuitively more reasonable direction.

Chi square goodness-of-fit tests on the observed and expected frequencies of "yes" responses ( $\mathrm{N}=$ 360 or 120) indicated an excellent fit of the multiplicative probability rule to the data. For $10, x^{2}=$ $8.8(\mathrm{df}=8, \mathrm{p}>\mathrm{.30})$; for $\mathrm{BW}$ and $\mathrm{JM}, \mathrm{X}^{2}=13$ ( $\mathrm{df}=6$, $p>.04)$. Furthermore, the absolute differences between observed and expected probabilities are very small, as shown in Table 3. All in all, there is really little question that the decision rule for disjunctive visual detection of widely spaced dots is the multiplicative probability rule, which combines decisions about the dots rather than combining the strengths of the dots.

\section{References}

Bouman, M. A., \& van den Brink, G. On the integrate capacity in time and space of the human peripheral retina. $J$. Opt. Soc. Amer. $1952,42,617-620$.

Casperon, R. C., \& Schlosberg, H. Monocular and binocular intensity thresholds for fields containing 1-7 dots. J. exp. Psychol. $1950,40,81-92$.

Collier, G. Probability of response and intertrial association as functions of monocular and binocular stimulation. $J$. exp. Psychol, , 1954, 47, 75-83.

Egan, J. P., Schulman, A. I., \& Greenberg, G. Z. Operating characteristics determined by binary decisions and by ratings. $J$ Acoust. Soc. Amer., 1959, 31, 768-773.

Green, D. M., \& Swets, J. A. Signal detection theory and psychophysics. New York: Wiley, 1966.

Luce, R. D. Detection and recognition. In R. D. Luce, R. R. Bush \& E. Galanter (Eds.), Handbook of mathematical psychulooy. New York: Wiley, 1963, 1. Pp. 103-189.

Matin, L. Binocular summation at the absolute threshold of peripheral vision. J. Opt, Soc. Amer, 1962, 52, 1276-1286.

Nachmias, J., \& Steinman, R. M. Study of absolute visual detection by the rating-scale method. J. Opt. Soc. Amer., 1963, 53, 12061213.

Pirenne, M. H. Binocular and uniocular threshold of vision. Nature, $1943,152,698-699$.

Swets, J. A., Shipley, Elizabeth, F., McKey, Mary J., \& Green D. M. Multiple observations of signals in noise. J. Acoust. Soc. Amer., 1959, 31, 514-521.

Swets, J. A., Tanner, W. P., Jr., \& Birdsall, T. G. Decision processes in perception. Psychol. Rev., 1961, 68, 301-340.

van den Brink, G., Bouman, M. A. Variation of integrative actions in the retinal system: in adaptational phenomenor. $J$. Opt. Soc. Amer., 1954, 44, 616-620.

\section{Note}

1. This work was supported primarily by grant $\mathrm{MH} 08890-03$ from the National Institute of Mental Health, U. S. Public Health Service. Further aid was received from a National Aeronautics and Space Administration grant NsG 496, to Hans-Lukas Teuber.

(Accepted for publication March 29, 1967.) 